\title{
Dispersive Optical Bistability in Quantum Wells With Logarithmic Gain
}

\author{
Tania Vivero, José Manuel Rivas-Moscoso, Ana Pilar González-Marcos, and \\ José Antonio Martín-Pereda, Senior Member, IEEE
}

\begin{abstract}
We present an analytical model for studying optical bistability in semiconductor lasers that exhibit a logarithmic dependence of the optical gain on carrier concentration. Model results are shown for a Fabry-Pérot quantum-well laser and compared with the predictions of a commercial computer-aided design (CAD) software tool.
\end{abstract}

Index Terms-Optical bistability, logarithmic gain, quantumwell, optical communications, hysteresis, numerical modeling.

\section{INTRODUCTION}

$\mathbf{O}$ PTICAL BISTABILITY $(\mathrm{OB})$ and its applications to the fields of optical computing and processing have received widespread attention in the past decades [1]-[4]. Nonlinearity and optical feedback lie at the very core of the bistability concept, which, in general terms, refers to the existence of two different output states for the same input over a range of input values [2]. Depending on the nonlinearity, two types of $\mathrm{OB}$ can be distinguished: absorptive and dispersive. The nonlinearity responsible for absorptive bistability arises from the dependence of the absorption coefficient on input light intensity in a laser containing a saturable absorber. This type of bistability was first observed in a Fabry-Pérot semiconductor laser under external optical injection in the 1960s [1] and, decades later, demonstrated experimentally in a two-section distributed feedback (DFB) laser [5]. Absorptive bistability has been proved to take place both in the output power/current characteristic [6] and in the output/input power characteristic [7]. A deeper review on the subject of absorptive bistability in semiconductor laser amplifiers can be found in [8].

Dispersive bistability, on the other hand, arises from the intensity dependence of the refractive index, which causes the shift of the cavity frequency toward resonance with the input field. It was first predicted theoretically in semiconductor laser amplifiers (SLA) at an operating wavelength of $840 \mathrm{~nm}$ [9] and $820 \mathrm{~nm}[10]$ in 1983. Later on, dispersive bistability was also demonstrated in a Fabry-Pérot SLA at $1.3 \mu \mathrm{m}$ [11] and in an InGaAsP Fabry-Pérot Laser Amplifier at $1.5 \mu \mathrm{m}$ [12], [13].

In this paper, our efforts will be concentrated on the analysis of dispersive $\mathrm{OB}$ in transparent semiconductor lasers where ab-

Manuscript received October 27, 2009; revised January 18, 2010. Current version published April 14, 2010. This work was supported by the grant program TIC2003-04309 of the Ministerio de Ciencia e Innovación, Spain.

The authors are with the Department of Photonic Technology, Universidad Politécnica de Madrid, Ciudad Universitaria s/n, 28040 Madrid, Spain (e-mail: tania.vivero@tfo.upm.es).

Color versions of one or more of the figures in this paper are available online at http://ieeexplore.ieee.org.

Digital Object Identifier 10.1109/JQE.2010.2044974 sorptive bistability can be neglected. A logarithmic relation between the optical material gain and the carrier concentration will be employed in the laser model and the results of the analysis will be applied to a quantum-well laser device. As compared with bulk semiconductor materials, quantum wells possess superior characteristics, such as low threshold current [14], [15], low temperature dependence [16], long-wavelength operation [17], and nonlinear effects [18], that justify our choice. Lastly, this analysis will be contrasted with the results of a well-known commercial photonics software tool.

\section{ANALYSIS}

The most popular methods to study the nonlinear dynamics of a laser with external optical injection are based on Fabry-Pérot and rate equation approaches. Even though both models give identical results in the limiting case of no injected signal [19], their results differ increasingly with the increase of the injected signal level [19], [20]. In [21] some modifications were introduced in both methods to minimize these differences.

The Fabry-Pérot method is based on the traveling-wave equations of a semiconductor laser in a simple Fabry-Pérot configuration and is used to predict the spatiotemporal dynamics of the electromagnetic field in the laser cavity [12], [22].

The rate equation (RE) method is based on the analysis of first-order differential equations for the carrier density inside the laser cavity [19], [23], [24]. One disadvantage of this method is that it does not include the spectral characteristics of the cavity; however, it is expected to give better results for small signal applications and it is suitable to study the dynamic characteristics and modulation properties of injection-locked semiconductor lasers [25].

Built on both approaches, a convenient analytical method was developed in [12] and [26]. On the one hand, the Fabry-Pérot method facilitates the calculation of an appropriate mean optical intensity, obtained by averaging the axial intensity distributions within the Fabry-Perot cavity, to derive relations for the input and output intensities. On the other hand, the nonlinear refraction is determined by the magnitude of the average internal intensity via its effect on the electron concentration in the active medium (RE method). This model is a simple and efficient tool for the analysis of Fabry-Pérot laser amplifiers under the approximation of a linear relation between the material gain and the carrier concentration [27]

$$
g_{m}=a_{\operatorname{lin}}\left(n-n_{0}\right)
$$

where $a_{\text {lin }}$ is the linear gain coefficient, $n$ is the average carrier concentration in the cavity, and $n_{0}$ is the transparency carrier density. 


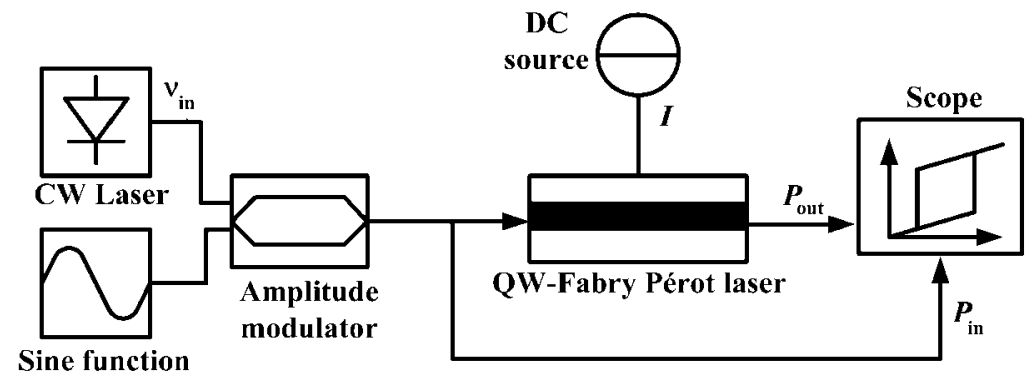

Fig. 1. Setup for the bistable operation of a quantum-well (QW) Fabry-Pérot laser.

However, various authors [28], [29] have pointed out that near threshold there exists a logarithmic relation between optical gain and carrier concentration, which, in a simplified form, can be expressed as

$$
g_{m}=a_{\log } \ln \left(n / n_{0}\right)
$$

where $a_{\log }$ is the logarithmic gain coefficient. This approximation is more accurate than the linear approximation used elsewhere [30], both in bulk [31] and in small quantum well lasers where the carrier population of higher levels is negligible [32], and therefore will be used, instead of the linear relation in (1), in the rest of the paper.

Let us focus on power OB in quantum-well Fabry-Pérot lasers for which the material-gain/carrier-concentration relation can be represented as indicated in (2). Fig. 1 shows a schematic of the setup for plotting the hysteresis loops. A constant-wave (CW) diode laser is amplitude-modulated by a sinusoidal electrical signal of several orders of magnitude lower frequency, and made incident upon a quantum-well Fabry-Pérot laser with an active region of reduced transversal dimensions, biased below threshold by a current $I=j w L$, where $j$ is the current density, and $w$ and $L$ are the active-region width and length, respectively. Output powers are plotted against input powers to observe the bistable behavior of the laser.

The evolution in time of the carrier concentration in the quantum well $(n)$ and the separate confinement heterostructure $(\mathrm{SCH})$ layer $\left(n_{\mathrm{SCH}}\right)$, and the change in photon density $(S)$ within the cavity are given by the rate equations [33]

$$
\begin{aligned}
\frac{\mathrm{d} n_{\mathrm{SCH}}}{\mathrm{d} t}= & \frac{j}{e d}-\frac{n_{\mathrm{SCH}}}{\tau_{c}}+\frac{n}{\tau_{e}} \frac{d}{d_{\mathrm{SCH}}}, \\
\frac{\mathrm{d} n}{\mathrm{~d} t}= & \frac{n_{\mathrm{SCH}}}{\tau_{c}} \frac{d_{\mathrm{SCH}}}{d}-n\left(\frac{1}{\tau_{e}}+\frac{1}{\tau}\right) \\
& -\frac{\Gamma c}{N \Delta E} \int_{0}^{\Delta E} g_{m}(E) S(z, E) \mathrm{d} E, \\
\frac{\mathrm{d} S}{\mathrm{~d} t}= & \frac{\Gamma c}{N \Delta E} \int_{0}^{\Delta E} g_{m}(E) S(z, E) \mathrm{d} E-\frac{S}{\tau_{p}},
\end{aligned}
$$

where $e$ is the electron charge, $d$ is the active-region thickness, $\tau_{c}$ is the carrier capture time from the SCH layer to the quantum well, $\tau_{e}$ is the carrier escape time from the quantum well to the $\mathrm{SCH}$ layer, $d_{\mathrm{SCH}}$ is the thickness of one side of the $\mathrm{SCH}$ region, $\tau$ is the electron lifetime, $\Gamma$ is the quantum-well confinement factor, $\Delta E$ is the gain-curve spectral width, $g_{m}$ is the material gain, $E$ is the photon energy, $c$ is the velocity of light, $\tau_{p}$ is the photon lifetime, and $N$ is the group effective refractive index of the cavity.

For the region of interest for bistable operation, the input light power is much larger than the spontaneous emission power generated within the device over one mode spacing centered on the input wavelength [12] and, therefore, the spontaneous emission coefficient can be neglected. We shall also neglect the dependence of the spontaneous emission rate on position $z$, the dependence of the net gain per unit length $g$ on position $z$ and photon energy, and the dependence of the photon density on photon energy for the modes of interest [26]. With these assumptions in place and bearing in mind that $S=P N /($ Ecwd $)$, the position-independent mean optical power, $P_{\text {av }}$, obtained by averaging the positive and negative traveling signal waves over a complete round trip of the cavity, and the input and output powers, $P_{\text {in }}$ and $P_{\text {out }}$, respectively, are given by [12]

$$
\begin{aligned}
P_{\mathrm{av}} & =\frac{\left(1-R_{1}\right)\left(1+R_{2} e^{g L}\right)\left(1-e^{-g L}\right)\left[P_{\mathrm{in}} /(g L)\right]}{\left(1-\sqrt{R_{1} R_{2}} e^{g L}\right)^{2}+4 \sqrt{R_{1} R_{2}} e^{g L} \sin ^{2}(\phi)}, \\
P_{\text {in }} & =\frac{\left(1-\sqrt{R_{1} R_{2} e^{g L}}\right)^{2}+4 \sqrt{R_{1} R_{2}} e^{g L} \sin ^{2}(\phi) P_{\mathrm{out}}}{\left(1-R_{1}\right)\left(1-R_{2}\right) e^{g L}}, \\
P_{\text {out }} & =\frac{\left(1-R_{2}\right) g L P_{\mathrm{av}}}{\left(1+R_{2} e^{g L}\right)\left(1-e^{-g L}\right)},
\end{aligned}
$$

where $R_{1}$ and $R_{2}$ are the reflectivities at the front and back cavity mirrors, $g$ is the net gain given by [26]

$$
g L=\Gamma g_{m} L-\alpha L
$$

and $\phi$ is the relative phase change of the signal as it passes through the cavity [34]

$$
\phi=\phi_{0}+\frac{2 \pi L}{\lambda} \Gamma\left(n-n_{1}\right) \frac{\mathrm{d} N}{\mathrm{~d} n}
$$

where $\phi_{0}$ can be written as

$$
\phi_{0}=\pi \frac{\nu_{\text {in }}-\nu^{F P}}{\Delta \nu}
$$

$\lambda$ is the operation wavelength, and $n_{1}$ is the carrier concentration in absence of optical power injection, given by $n_{1}=j \tau /(e d)$.

The initial phase detuning $\phi_{0}$, as stated by (11), is a function of the frequency difference between the input signal and the nearest Fabry-Pérot modes. The spectral positions of the laser modes vary with the bias current applied to the laser and the input power, the phase separation between two adjacent modes being $\pi$. As can be seen in Fig. 2, power injection provokes a 


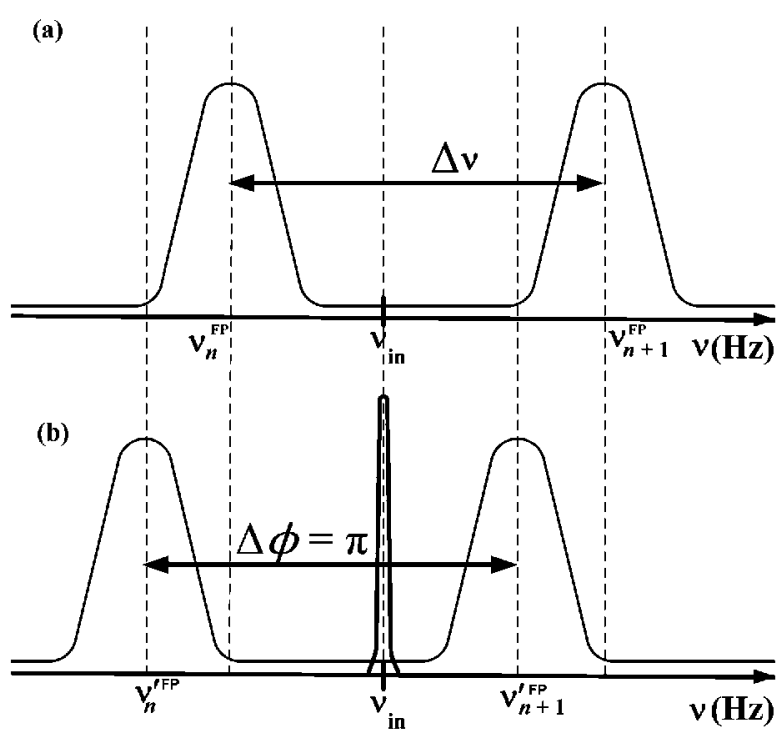

Fig. 2. (a) Fabry-Pérot modes in a quantum-well laser in absence of power injection. (b) The modes shift when an optical signal is incident upon the cavity.

displacement of the Fabry-Pérot modes toward lower frequencies, which results in a variation of the output signal phase in a quantity related to the derivative of phase $\phi$ in (10) with respect to carrier concentration $n$

$$
\frac{\mathrm{d} \phi}{\mathrm{d} n} \approx \frac{2 \pi L}{\lambda} \Gamma \frac{\mathrm{d} N}{\mathrm{~d} n}
$$

Using the average power given by (6) and multiplying the addition of (3) and (4) by $\tau$, the rate equations reduce to

$$
\tau\left(\frac{\mathrm{d} n}{\mathrm{~d} t}+\frac{\mathrm{d} n_{\mathrm{SCH}}}{\mathrm{d} t} \frac{d_{\mathrm{SCH}}}{d}\right)=n_{1}-n-\frac{\Gamma g_{m} \tau}{E w d} P_{\mathrm{av}}
$$

which, after multiplication by $(\mathrm{d} \phi) /(\mathrm{d} n)$ and the use of (2), yield

$$
\begin{aligned}
\left(1+\frac{\mathrm{d} n_{\mathrm{SCH}}}{\mathrm{d} n} \frac{d_{\mathrm{SCH}}}{d}\right) & \tau \frac{\mathrm{d} \phi}{\mathrm{d} t}=\left(n_{1}-n\right) \frac{2 \pi L}{\lambda} \Gamma \frac{\mathrm{d} N}{\mathrm{~d} n} \\
& -\frac{\Gamma}{E} I_{\mathrm{av}} \tau a_{\log } \ln \left(\frac{n}{n_{0}}\right) \frac{2 \pi L}{\lambda} \Gamma \frac{\mathrm{d} N}{\mathrm{~d} n}
\end{aligned}
$$

where $n_{0}$ is the transparency carrier density.

Because the relative magnitudes of the round-trip time in the cavity $\tau_{g}$ and the average lifetime of the carriers $\tau$ encountered in the semiconductors of interest are such that $\tau_{g} \ll \tau$, the derivative $(\mathrm{d} \phi) /(\mathrm{d} t)$ in (14) can be neglected [35]. Likewise, the natural logarithm $\ln \left(n / n_{0}\right)$ in (14) admits a Taylor series expansion around $n \approx n_{1}$

$$
\ln \left(\frac{n}{n_{0}}\right)=\ln \left(\frac{n_{1}}{n_{0}}\right)+\frac{n-n_{1}}{n_{1}}-\frac{1}{2} \frac{\left(n-n_{1}\right)^{2}}{n_{1}^{2}}
$$

that, in conjunction with (10), allows writing (14) as

$$
\begin{aligned}
& \left(\phi_{0}-\phi\right)=\frac{\Gamma^{2}}{E} a_{\log } \frac{2 \pi L \tau P_{\mathrm{av}}}{\lambda w d} \frac{\mathrm{d} N}{\mathrm{~d} n} \ln \left(\frac{n_{1}}{n_{0}}\right) \\
& +\frac{\Gamma a_{\log } \tau P_{\mathrm{av}}}{E n_{1} w d}\left(\phi-\phi_{0}\right)-\frac{a_{\log } P_{\mathrm{av}} \tau \lambda}{4 \pi L^{2} n_{1}^{2} E w d} \frac{\mathrm{d} n}{\mathrm{~d} N}\left(\phi-\phi_{0}\right)^{2}
\end{aligned}
$$

and, consequently, the phase change $\phi$ of the signal as it passes through the cavity is finally given by

$$
\begin{aligned}
& \phi=\phi_{0}+ \frac{2 \pi L n_{1} \Gamma}{\lambda} \frac{\mathrm{d} N}{\mathrm{~d} n}\left\{1+\frac{E n_{1}}{\Gamma a_{\log } P_{\mathrm{av}} \tau}\right. \\
&\left. \pm\left[\left(\frac{E n_{1}}{\Gamma a_{\log } P_{\mathrm{av}} \tau}+1\right)^{2}+2 \ln \left(\frac{n_{1}}{n_{0}}\right)\right]^{\frac{1}{2}}\right\} .
\end{aligned}
$$

Using (2), (10) and (15), the net gain given by (9) can be written as

$$
\begin{aligned}
g L=\Gamma a_{\log } L \ln \left\{\frac{n_{1}}{n_{0}}\right. & {\left[\frac{\lambda}{2 \pi L \Gamma n_{1}}\right.} \\
& \left.\left.\times\left(\frac{\mathrm{d} N}{\mathrm{~d} n}\right)^{-1}\left(\phi-\phi_{0}\right)+1\right]\right\}-\alpha L
\end{aligned}
$$

which, after substitution of (17) into (18), results in

$$
\begin{aligned}
g L= & \Gamma a_{\log } L \ln \left(\frac { n _ { 1 } } { n _ { 0 } } \left\{1+\frac{E n_{1}}{\Gamma a_{\log } P_{\mathrm{av}} \tau}\right.\right. \\
& \pm\left[\left(\frac{E n_{1}}{\Gamma a_{\log } P_{\mathrm{av}} \tau}+1\right)^{2}\right. \\
& \left.\left.\left.+2 \ln \left(\frac{n_{1}}{n_{0}}\right)\right]^{\frac{1}{2}}+1\right\}\right)-\alpha L .
\end{aligned}
$$

It is straightforward, though somewhat tedious, to verify that (17) and (19), with the minus sign, reduce to the equations for the linear gain model

$$
\begin{aligned}
g L & =\frac{\Gamma a_{\log }\left(n_{1}-n_{0}\right) L}{n_{0}\left(1+P_{\mathrm{av}} / P_{s}\right)}-\alpha L \\
\phi & =\phi_{0}+\frac{\Gamma a_{\log }\left(n_{1}-n_{0}\right) L b}{2 n_{0}} \frac{P_{\mathrm{av}} / P_{s}}{\left(1+P_{\mathrm{av}} / P_{s}\right)}
\end{aligned}
$$

where $P_{s}=n_{0} h \nu w d /\left(a_{\log } \Gamma \tau\right)$ and $b=-4 \pi n_{0} /\left(\lambda a_{\log }\right)(\mathrm{d} N / \mathrm{d} n)$, in the limit when the carrier concentration tends to the transparency carrier density. For the sake of comparing the results obtained with the logarithmic model derived in this section and those given by the linear model in [12], Fig. 3 shows the variation of the phase change and the net gain in a Fabry-Pérot laser analyzed with both gain models for increasing carrier concentrations. As was pointed out, an increasing divergence of both curves can be observed as the difference between the carrier concentration and the transparency carrier density increases. This divergence is more remarkable in the region where the curves exhibit a bistable behavior, which makes the logarithmic model described in this section more relevant for the study of $\mathrm{OB}$ in Fabry-Pérot semiconductor lasers.

Equations (6), (7), (17) and (19) will be numerically solved to validate our logarithmic gain model with a view to the observation of power OB in a Fabry-Pérot quantum-well laser.

\section{NuMERICAL RESULTS}

Let us consider the equations in the previous section and the parameters listed in Table I for a Fabry-Pérot quantum-well laser operated in transmission. The electron lifetime is given by 


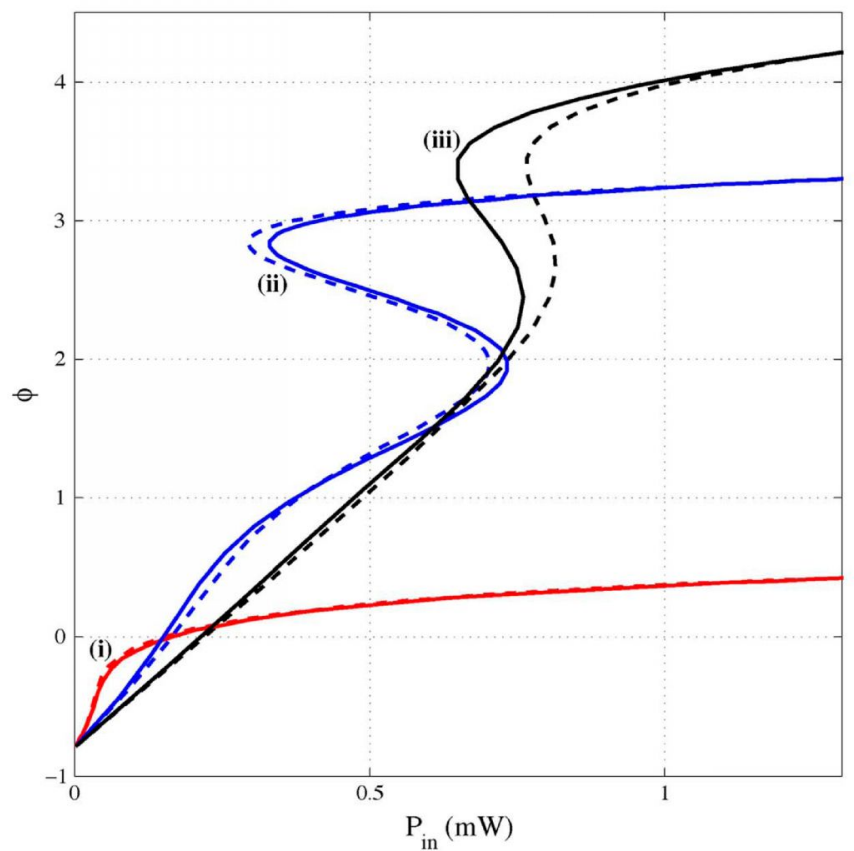

(a)

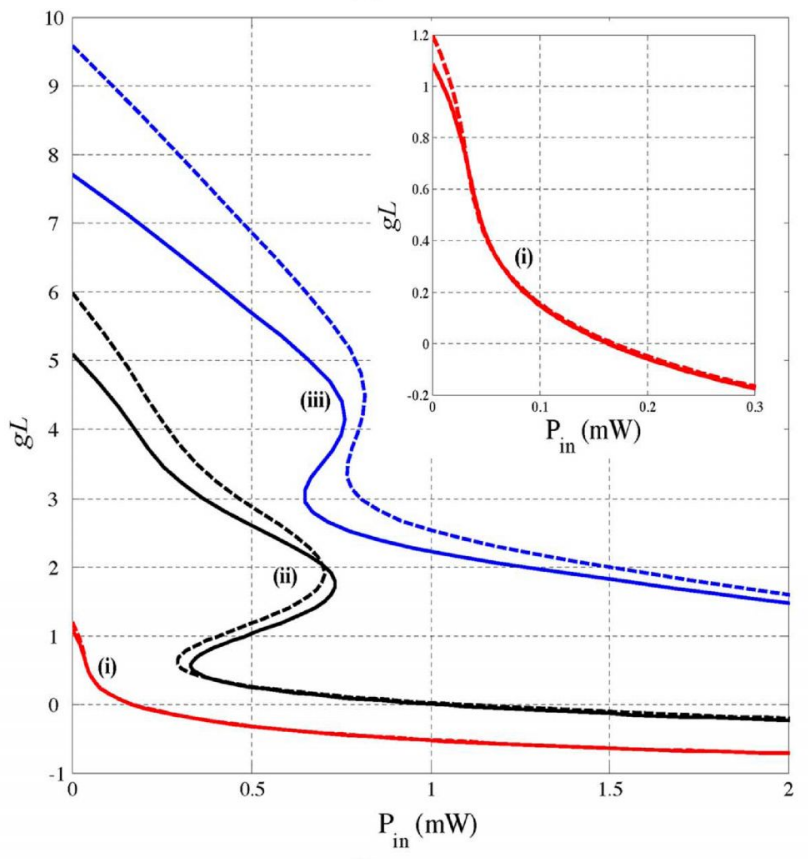

(b)

Fig. 3. (a) Phase change, $\phi$, and (b) net gain, $g L$, as a function of input power, $P_{\text {in }}$, for carrier concentrations in the absence of optical power, $n_{1}$, (i) $1.1 \times 10^{24}$ $\mathrm{m}^{-3}$, (ii) $1.3 \times 10^{24} \mathrm{~m}^{-3}$, and (iii) $1.45 \times 10^{24} \mathrm{~m}^{-3}$, obtained using a logarithmic gain model (solid line) and a linear gain model (dashed line). Data $\phi_{0}=-0.25 \pi, L=400 \mu \mathrm{m}, \Gamma=1.0, \alpha=3000, \nu=193.1 \mathrm{THz}$ $\mathrm{d} N / \mathrm{d} n=-1 \times 10^{-26} \mathrm{~m}^{3}, a_{\log }=6 \times 10^{4} \mathrm{~m}^{-1}, \tau=\left(A+B n_{1}+\right.$ $\left.C n_{1}^{2}\right)^{-1}, n_{0}=1.0 \times 10^{24} \mathrm{~m}^{-3}, w=2.5 \mu \mathrm{m}, d=200 \mathrm{~nm}$. In (b) an inset shows an enlargement for $n_{1}=1.1 \times 10^{24} \mathrm{~m}^{-3}$.

$\tau=\left(A+B n_{1}+C n_{1}^{2}\right)^{-1}$, where $A, B$ and $C$ are the linear, bimolecular and Auger recombination coefficients, respectively, whose values are shown in Table I. A difference in definition between $a_{\log }$ and $a_{\text {lin }}$ is responsible for the difference in order of magnitude between the value reported in Table I and the usual values for $a_{\operatorname{lin}}$ [36]. In order for $a_{\log }$ to have a value in the same
TABLE I

QUANTUM-Well LASER PARAMETERS

\begin{tabular}{clcc}
\hline \hline Symbol & Parameter & Value & Units \\
\hline$L$ & Device section length & 400 & $\mu \mathrm{m}$ \\
$w$ & Active region width & 2.5 & $\mu \mathrm{m}$ \\
$d$ & Active region thickness & 40 & $\mu \mathrm{m}$ \\
$\lambda$ & Nominal wavelength & 1.5525 & $\mu \mathrm{m}$ \\
$N$ & Effective index in the cavity & 3.7 & \\
$R$ & Left/right facet reflectivities & 0.32 & \\
$\Gamma$ & QW Confinement factor & 0.05 & \\
$a_{\log }$ & Logarithmic material gain coefficient & $6 \times 10^{4}$ & $\mathrm{~m}^{-1}$ \\
$\mathrm{~d} N / \mathrm{d} n$ & QW Differential index & $-1 \times 10^{-26}$ & $\mathrm{~m}^{3}$ \\
$A$ & Linear recombination coefficient & 0 & $\mathrm{~s}^{-1}$ \\
$B$ & Bimolecular recombination coefficient & $2.2 \times 10^{-16}$ & $\mathrm{~m}^{3} \mathrm{~s}^{-1}$ \\
$C$ & Auger recombination coefficient & $5.24 \times 10^{-41}$ & $\mathrm{~m}^{6} \mathrm{~s}^{-1}$ \\
$\alpha$ & Internal loss & 3000 & $\mathrm{~m}^{-1}$ \\
$n_{0}$ & Transparency carrier density & $1 \times 10^{24}$ & $\mathrm{~m}^{-3}$ \\
\hline \hline
\end{tabular}

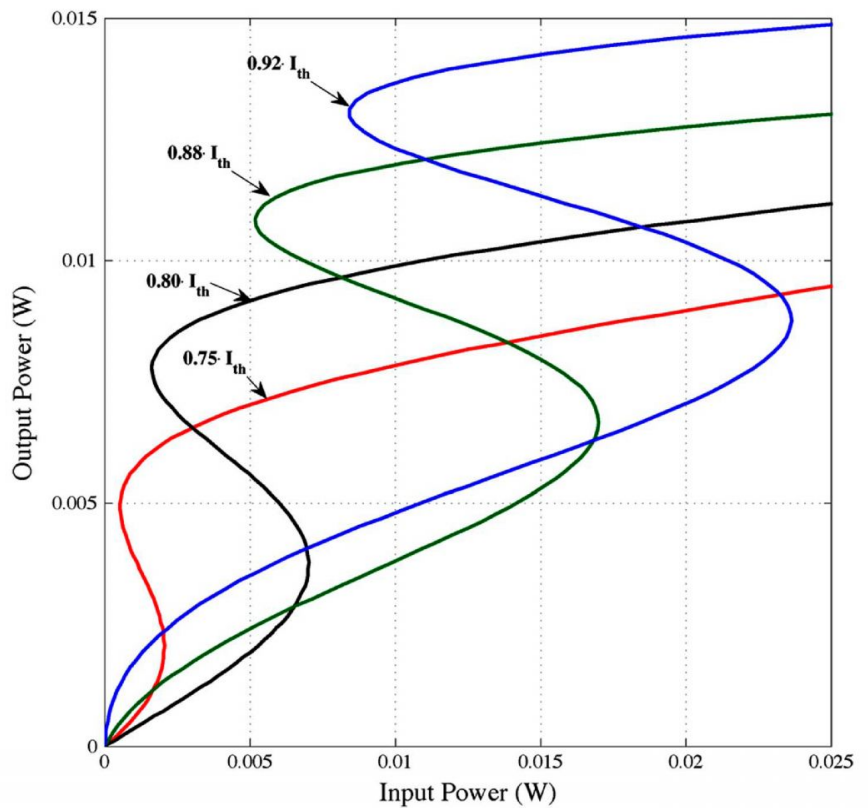

Fig. 4. Output power of the Fabry-Pérot quantum-well laser for several bias currents.

order of magnitude as $a_{\text {lin }}$, (2) must be multiplied by $n_{0}$, such that $a_{\log }$ will assume an $n_{0}$-times lower value.

The laser was biased at $75 \%, 80 \%, 88 \%$ and $92 \%$ of the threshold current, which, with the above parameters, is given by $\mathrm{I}_{\mathrm{th}}=188 \mathrm{~mA}$, and the corresponding initial detuning was $-0.32 \pi,-0.52 \pi,-0.80 \pi$ and $-0.95 \pi$, respectively. As illustrated in Fig. 4, hysteresis loops in an anticlockwise direction [37] in the quantum-well laser can be observed in the optical output-input characteristic.

We notice that, as the dc bias is increased, the optical input requirement is higher in order to achieve $\mathrm{OB}$. The output power is also higher. Likewise, the amount of hysteresis increases with increasing bias currents. Finally, for input powers above the power at which the output signal jumps from a low level to a high level (i.e., outside the hysteresis cycle), gain at the upper state is only achieved for currents $0.75 \cdot \mathrm{I}_{\text {th }}$ and $0.80 \cdot \mathrm{I}_{\text {th }}$. This is a consequence of the fact that, for increasing input powers, 
amplification is only obtained for bias currents near the laser threshold and for initial phase detunings $\left|\phi_{0}\right| \leq 0.70 \pi$.

\section{COMPuTATIONAL MODEL}

The schematic shown in Fig. 1 was also simulated with commercial software tool VPItransmissionMaker ${ }^{\mathrm{TM}}$ version 8.0 by VPIsystems $^{\mathrm{TM}}$ [38]. In VPItransmissionMaker ${ }^{\mathrm{TM}}$ lasers are analyzed using the transmission-line laser model (TLLM), whereby the laser cavity is divided into small longitudinal sections, each section being divided in turn into scattering and transmission nodes, connected by so-called transmission lines, which represent the waveguide propagation delay [39], [40].

Based on the said schematic, we selected a CW DFB laser (LaserCW module) with an emission frequency of $193.1 \mathrm{THz}$ and an ideal linewidth of $0 \mathrm{~Hz}$ to feed an amplitude modulator (modulatorAM module) whose output power follows the amplitude of a $0.975-\mathrm{MHz}$ sine electrical signal (FuncSineEl module), chosen for convenience. The amplitude-modulated optical signal enters the bistable laser (LaserTLM module), a transmission-line model of a Fabry-Pérot laser with a multi-quantum well structure in the active region, described by the parameters given in Table I. A PowerMeter module with no bandwidth-limiting filter averages the light at the input and output faces of the laser module over time intervals of $6.4 \mathrm{~ns}$ and transfers both values to a NumericalAnalizer $2 D$ module for two-dimensional numerical data. After several runs of the simulation, a plot of the hysteresis loop of the laser can be obtained.

To compare the results of our model presented in Section III with the computational model being described in this section, two cases have been singled out: since amplification was only achieved for bias currents of $0.75 \cdot \mathrm{I}_{\text {th }}$ and $0.80 \cdot \mathrm{I}_{\mathrm{th}}$, only these two situations have been retained and considered. As was pointed out in the previous section, the corresponding initial phase detuning between the external injected signal and the resonance of the laser are $-0.32 \pi$ and $-0.52 \pi$, respectively. Also, because of the different power requirements of the Fabry-Pérot laser for different bias currents, average powers of $3 \mathrm{~mW}$ and $9 \mathrm{~mW}$, respectively, have been used for each of the two cases mentioned above.

As was shown in Fig. 2, the input signal incident upon the quantum-well laser affects the laser's response by inducing a change in the refractive index of the semiconductor material that leads to a change in the laser emission spectrum. This change manifests itself in a displacement of the emission modes toward lower frequencies. When, as a consequence of this mode shift, the frequency of the mode is coincident with that of the incident signal, there occurs a switch from a low power level to a high power level, or vice versa, at the output of the laser. On account of the fact that this switch takes place at a different power for increasing and decreasing input powers, a hysteresis loop appears in the optical output-input characteristic.

Fig. 5 shows the quantum-well laser output power represented as a function of the input power using the numerical model (a) and the computational model (b), for a bias current of $0.75 \cdot \mathrm{I}_{\mathrm{th}}$. In both plots, the bistable curves jump to the ON state for input powers higher than $2 \mathrm{~mW}$, and return to the OFF state when $\mathrm{P}_{\text {in }}$ descends below $0.5 \mathrm{~mW}$. The output signal switches between

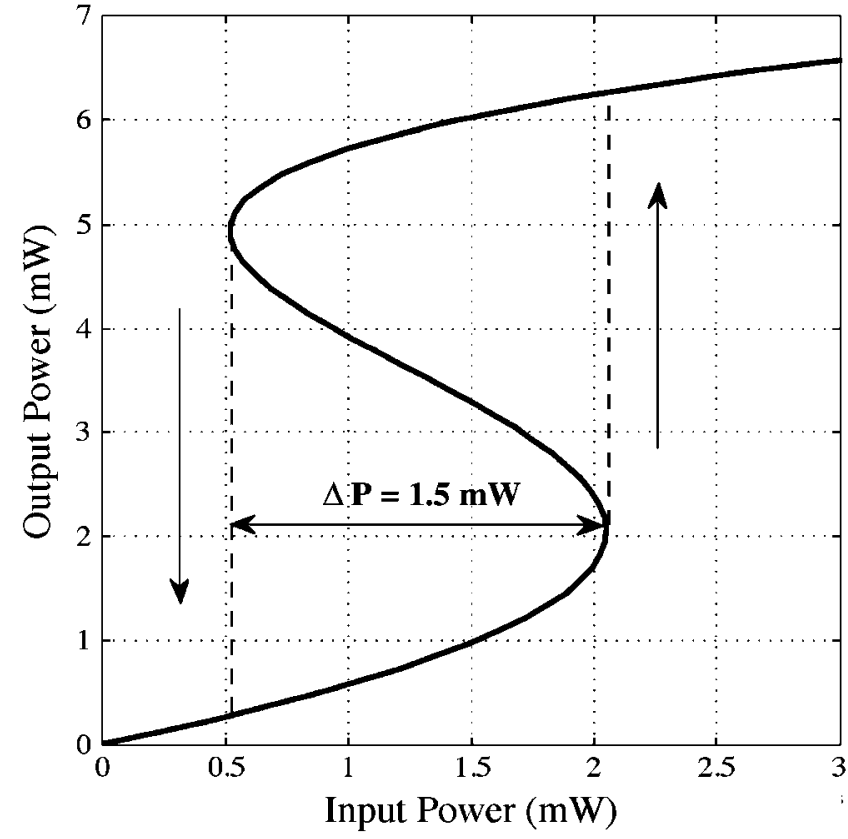

(a)

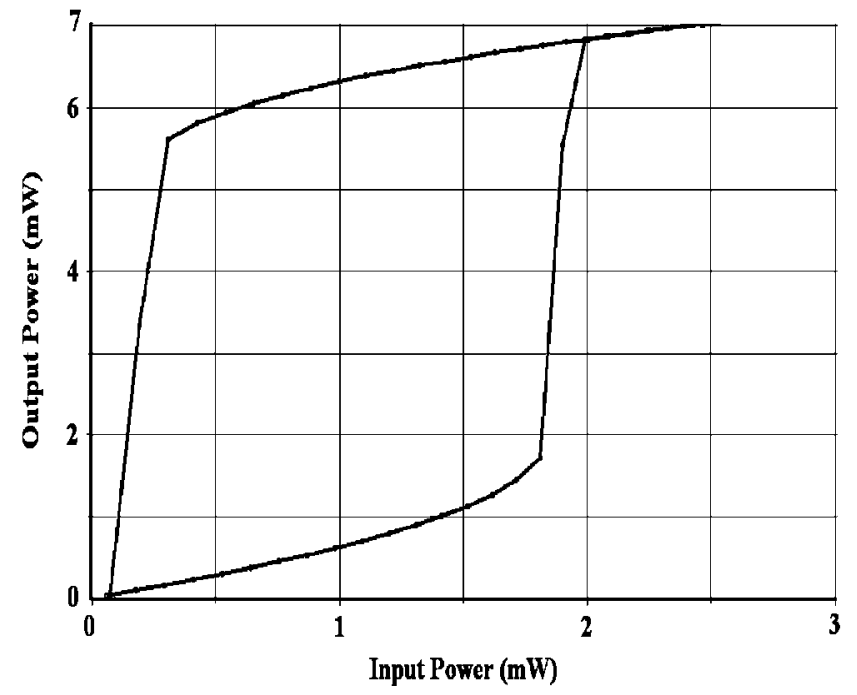

(b)

Fig. 5. (a) Numerical and (b) computational simulation for $0.75 \cdot I_{t h}$

those two states with average powers around $5.5 \mathrm{~mW}$ and $1 \mathrm{~mW}$. As we can see, there is good agreement between both models.

Fig. 6 shows in turn the bistable curves for a bias current of $0.80 \cdot \mathrm{I}_{\mathrm{th}_{1}}$. The curves move to the upper state at $\mathrm{P}_{\mathrm{in}}=7 \mathrm{~mW}$, attaining a $P_{\text {out }}=10 \mathrm{~mW}$, and drop back to the OFF state when $P_{\text {in }}=2 \mathrm{~mW}$ (with the model described in this paper) or 0.22 $\mathrm{mW}$ (with the computational simulation). The theoretical plot correctly determines the switch-on input power as compared with the simulation results, but there is a discrepancy in the prediction of the switch-off power, which is significantly lower with VPItransmissionMaker ${ }^{\mathrm{TM}}$. Output power results are, however, coincident with both models.

\section{CONClusion}

Existing models of semiconductor laser amplifiers, originally developed for studying materials with a linearly varying gain 


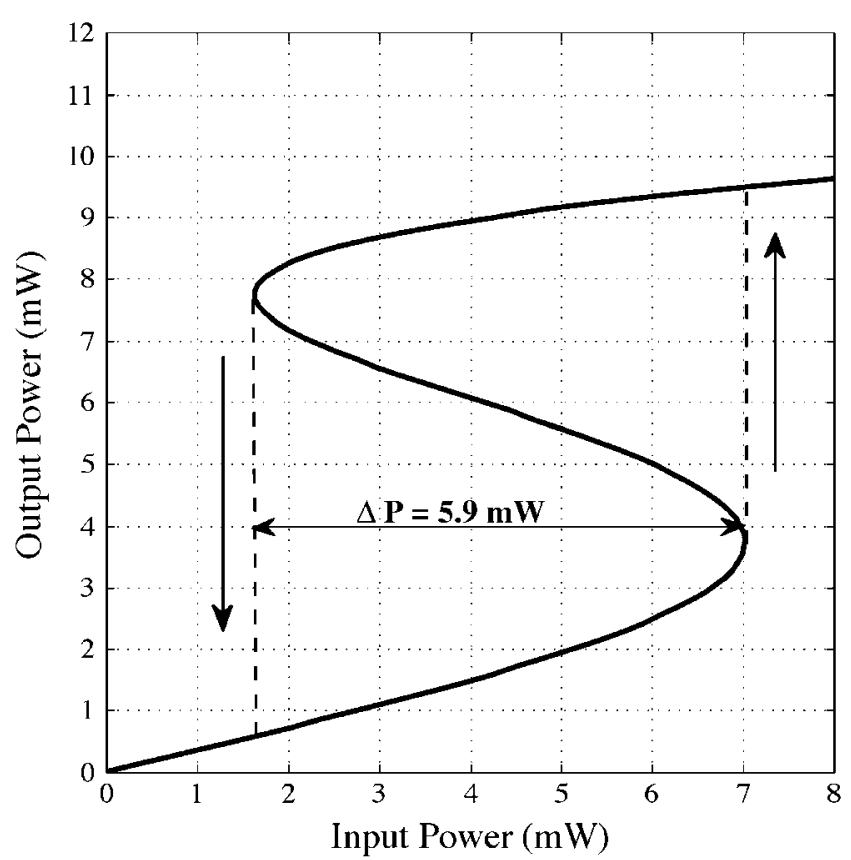

(a)

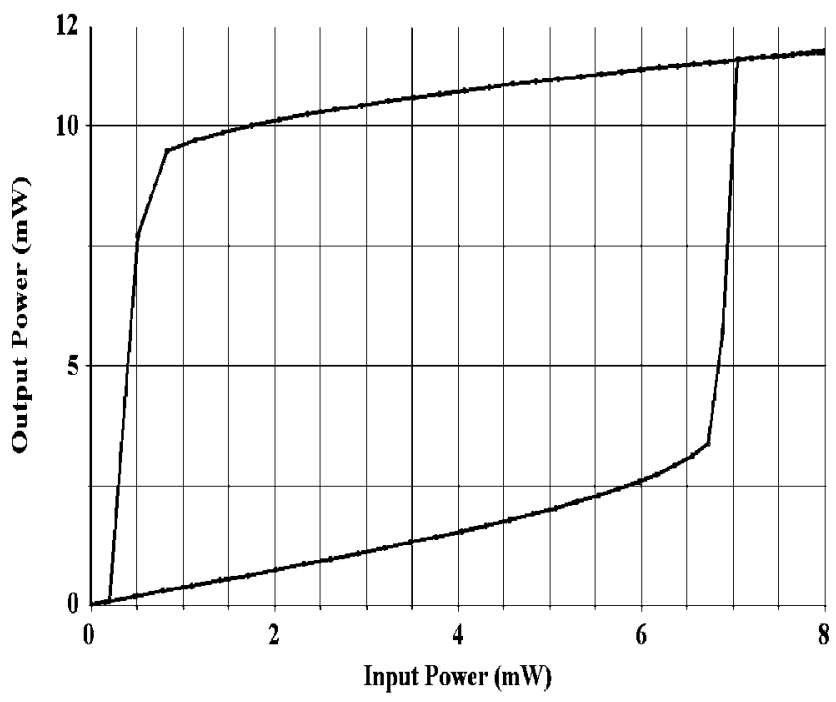

(b)

Fig. 6. (a) Numerical and (b) computational simulation for $0.80 \cdot \mathrm{I}_{1 \mathrm{~h}}$.

with carrier concentration, were reviewed and a new model was proposed to account for a logarithmic material-gain/carrier-concentration relation, which has been found to be a more accurate approximation in bulk and quantum-well semiconductors [31], [32]. Based on this analysis, dispersive OB in Fabry-Pérot quantum-well lasers was investigated and hysteresis loops in an anticlockwise direction in the output-input power characteristic were observed. Good agreement between the results obtained from the theoretical model presented in this paper and commercial software tool VPItransmissionMaker ${ }^{\mathrm{TM}}$ was found, both with regard to output power levels and with regard to switch-on powers. However, an increasing divergence in the determination of the switch-off power with increasing bias currents was also observed. In spite of this, the model dealt with in this paper, owing to its simplicity and reasonable accuracy, has proved to be a good tool to study $\mathrm{OB}$ in Fabry-Pérot semiconductor lasers. An experimental study to assess the accuracy of the logarithmic model should be carried out in the future due to the lack, to the best of our knowledge, of published experimental results in line with this work.

\section{REFERENCES}

[1] G. Lasher, "Analysis of a proposed bistable injection laser," Solid State Electron., vol. 7, pp. 707-716, Oct. 1964

[2] H. M. Gibbs, Optical Bistability: Controling Light With Light. New York, NY: Academic Press, 1985.

[3] A. P. González-Marcos and J. A. Martín-Pereda, "Photonic switching architectures with logic cells," in Proc. SPIE. Optical Transmission Systems and Equipment for WDM Networking 11 , Orlando, FL, 2003, pp. 569-579

[4] A. P. González-Marcos and J. A. Martín-Pereda, "Optically programmable logic cells," in Proc. SPIE, Photonic Devices and Algorithms for Computing III, Seattle, WA, 2001, pp. 65-73.

[5] H. Kawaguchi, K. Magari, H. Yasaka, M. Fukuda, and K. Oe, "Tunable optical-wavelength conversion using an optically triggerable multielectrode distributed feedback laser diode," IEEE J. Quantum Electron., vol. 24 , pp. 2153-2159, Nov. 1988 .

[6] H. Kawaguchi and G. Iwane, "Bistable operation in semiconductor lasers with inhomogeneous excitation," Electron. Lett., vol. 17, pp 167-168, Feb. 1981.

[7] H. Kawaguchi, "Bistable operation of semiconductor lasers by optical injection," Electron. Lett., vol. 17, pp. 741-742, Oct. 1981.

[8] H. Kawaguchi, "Absorptive and dispersive bistability in semiconductor injection lasers," Opt. Quantum Electron., vol. 19, pp. S1-S36, 1987.

[9] K. Otsuka and H. Iwamura, "Analysis of a multistable semiconductor light amplifier," IEEE J. Quantum Electron., vol. QE-19, pp. 1184-1186, Jul. 1983.

[10] T. Nakai, N. Ogasawara, and R. Itoa, "Optical bistability in a semiconductor laser amplifier," Jpn. J. Appl. Phys., vol. 22, pp. 310-312, 1983.

[11] W.F. Sharfin and M. Dagenaisa, "Room-temperature optical bistability in InGaAsP/InP amplifiers and implications for passive devices," Appl. Phys. Lett., vol. 46, pp. 819-821, May 1985.

[12] M. J. Adams, H. Westlake, M. O'Mahony, and I. Henning, "A comparison of active and passive optical bistability in semiconductors," IEEE J. Quantum Electron., vol. 21, pp. 1498-1504, Sep. 1985.

[13] H. J. Westlake, M. J. Adams, and M. J. OMahony, "Measurement of optical bistability in an InGaAsP laser amplifier at $1.5 \mu \mathrm{m}, "$ Electron. Lett., vol. 21, pp. 992-993, Oct. 1985.

[14] W. T. Sang, "Extremely low threshold (AIGa)As graded-index waveguide separate-confinement heterostructure lasers grown by molecular beam epitaxy," Appl. Phys. Lett., vol. 40, pp. 217-219, Feb. 1982.

[15] R. D. Burnham, W. Streifer, D. R. Scifres, C. Lindstrom, T. L. Paoli, and N. Holonyak, Jr., "Low-threshold single quantum well $(60 \mathrm{~A})$ GaAlAs lasers grown by MO-CVD with $\mathrm{Mg}$ as p-type dopant," Electron. Lett, vol. 18, pp. 1095-1097, Dec. 1982.

[16] R. Chin, N. Holonyak, Jr., B. A. Vojak, K. Hess, R. D. Dupuis, and P. D. Dapkus, "Temperature dependence of threshold current for quantumwell $\mathrm{Al}_{x}, \mathrm{Ga}_{1-x}$ As-GaAs heterostructure laser diodes," Appl. Phys Lett., vol. 36, pp. 19-21, Jan. 1980.

[17] H. Temkin, K. Alavi, W. R. Wagner, T. P. Pearsall, and A. Y. Cho, "1.5-1.6 $\mu \mathrm{m} \mathrm{Ga} \mathrm{Ga}_{0.47} \operatorname{In}_{0.53} \mathrm{As} / \mathrm{Al}_{0.48} \operatorname{In}_{0.52} \mathrm{As}$ multiquantum well lasers grown by molecular beam epitaxy," Appl. Phys. Lett., vol. 4323, pp. 845-847, May 1983.

[18] D. Chemla, D. Miller, P. Smith, A. Gossard, and W. Wiegmann, "Room temperature excitonic nonlinear absorption and refraction in GaAs/AlGaAs multiple quantum well structures," IEEE J. Quantun Electron., vol. 20 , pp. 265-275, Mar. 1984

[19] M. M. Ibrahim and M. S. Ibrahim, "A comparison between rate-equation and Fabry-Perot amplifier models of injection locked laser diodes," Opt. Laser Technol, vol. 28, pp. 39-42, Feb. 1996.

[20] R. Gordon, "Fabry-Perot semiconductor laser injection locking," IEEE J. Quantum Electron., vol. 42, pp. 353-356, Apr. 2006.

[21] D. Labukhin, C. A. Stolz, N. A. Zakhleniuk, R. Loudon, and M. J. Adams, "Modified Fabry-Perot and rate equation methods for the nonlinear dynamics of an optically injected semiconductor laser," IEEE $J$. Quantum Electron., vol. 45, pp. 863-871, July 2009.

[22] M. J. O'Mahony, "Semiconductor laser optical amplifiers for use in future fiber systems," J. Lightw. Technol., vol. 6, pp. 531-544, Apr. 1988 . 
[23] K. E. Chlouverakis and M. J. Adams, "Stability maps of injection-locked laser diodes using the largest Lyapunov exponent," Opt Commun., vol. 216, p. 405, Feb. 2003.

[24] R. Lang, "Injection locking properties of a semiconductor-laser," IEEE J. Quantum Electron., vol. QE-18, p. 976, Jun. 1982.

[25] O. Lidoyne and P. B. Gallion, "Modulation properties of an injectionlocked semiconductor laser," IEEE J. Quantum Electron., vol. 27, pp 344-351, 1991.

[26] M. J. Adams, J. V. Collins, and I. D. Henning, "Analysis of semiconductor laser optical amplifiers," IEE J. Optoelectron., vol. 132, pp. 58-63, Feb. 1985.

[27] H. Kawaguchi, Bistabilities and Nonlinearities in LaserDiodes. Norwood, MA: Artech House, 1994

[28] T.-A. Ma, Z.-M. Li, T. Makino, and M. S. Wartak, "Approximate optical gain formulas for $1.55-\mu \mathrm{m}$ strained quaternary quantum-well lasers," IEEE. J. Quantum Electron., vol. 31, pp. 29-34, Jan. 1995.

[29] J. Z. Wilcox, G. L. Peterson, S. Ou, J. J. Yang, and M. Jansen, "Gain and threshold-current dependence for multiple-quantum-well lasers,' J. Appl. Phys., vol. 64, pp. 6564-6569, Dec. 1988.

[30] T. A. DeTemple and C. M. Herzinger, "On the semiconductor laser logarithmic gain-current density relation," IEEE J. Quantum Electron., vol. 29, pp. 1246-1252, May 1993.

[31] Y. Barbarin, E. Bente, G. Servanton, L. Mussard, Y. Oei, R. Ntzel and M. Smit, "Gain measurements of Fabry-Perot InP/InGaAsP lasers using an ultrahigh-resolution spectrometer," Appl. Opt., vol. 45, pp. 9007-9012, 2006.

[32] P. M. Ilroy, A. Kurobe, and Y. Uematsu, "Analysis and application of theoretical gain curves to the design of multi-quantum-well lasers," IEEE J. Quantum Electron., vol. 21, pp. 1958-1963, Dec. 1985.

[33] R. Nagarajan, M. Ishikawa, T. Fukushima, R. S. Geels, and J. E. Bowers, "High speed quantum-well lasers and carrier transport effects," IEEE J. Quantum Electron, vol. 28, no. 10, pp. 1990-2008, Oct. 1992.

[34] A. J. Lowery, "A qualitative comparison between two semiconductor laser amplifier equivalent circuit models," IEEE J. Quantum Electron., vol. 26, pp. 1369-1375, Aug. 1990.

[35] M. J. Adams, "Time dependent analysis of active and passive optical bistability in semiconductors," IEE J. Optoelectron., vol. 132, pp. 343-348, Dec. 1985.

[36] Y. A. Goldberg and N. M. Schmidt, Handbook Series on Semiconductor Parameters. Singapore: World Scientific, 1999.

[37] P. Pakdeevanich and M. J. Adams, "Measurements and modeling of reflective bistability in 1.55-m laser diode amplifiers," IEEE J. Quantum Electron., vol. 35, pp. 1894-1903, Dec. 1999.

[38] VPI. Photonics, [Online]. Available: http://www.vpiphotonics.com, Available

[39] A. J. Lowery, "New dynamics semiconductor laser model based on the transmission-line modeling method," in IEE Proc. Optoelectron., Oct. 1987, vol. 134, pp. 281-289.
[40] W. J. R. Hoefer, "The transmission-line matrix method-Theory and applications," IEEE Trans. Microw. Theory Tech., vol. 33, pp. 882-893, 1985.

Tania Vivero received the B.S. degree in electronic engineering from the Universidad Católica Santa María La Antigua, Panama, in 2002, and the M.S. degree in software engineering from the Universidad Pontificia de Salamanca, Spain, in 2005. She is currently working toward the Ph.D. degree at the Universidad Politécnica de Madrid, Spain.

Her research involves optical bistability, semiconductor laser amplifiers, and optical networks.

José Manuel Rivas-Moscoso received the B.S., M.S. and Ph.D. degrees in physics from the Universidad de Santiago de Compostela, Spain, in 1998, 1999, and 2004 , respectively.

He worked as a Postdoctoral Researcher at the University of Cambridge, Cambridge, U.K., in 2005 and 2006, and has been with the Universidad Politécnica de Madrid, Spain, since 2007.

Ana Pilar González-Marcos received the Ph.D. degree from E.T.S. Ingenieros de Telecomunicación, Universidad Politécnica de Madrid (UPM), Spain, in 1993.

She was previously with private companies, where she was involved in CAD-CAE topics. She was an Assistant Professor with the Universidad de Alcalá de Henares, Spain, and an Associate Professor with the Universidad Alfonso X El Sabio, Spain. Since 1996, she has been a Professor with the E.T.S. Ingenieros de Telecomunicación, UPM. Her present interests are optical computing, the applications of chaos theory to optical communications, and the modeling of mammalian retina.

José Antonio Martín-Pereda (S'63-M'70-SM'91) received the Ph.D. degree from E.T.S. Ingenieros de Telecomunicación, Universidad Politécnica de Madrid (UPM), Spain, in 1971.

He has been a full Professor with UPM since 1975 and was a member of several technical programme conferences, mainly in optical communications (ECOC, OFC, IOOC) from 1985 until 1994. He has published more than 100 papers in journals and books and was the recipient of several academic and technical prizes. His present interest is the study of biological systems on models for artificial systems.

Prof. Martín-Pereda is a member of the Royal Spanish Academy of Engineering, the Optical Society of America, and SPIE. 